\title{
PREVISÕES PARA O CRESCIMENTO DO PIB TRIMESTRAL BRASILEIRO COM SÉRIES FINANCEIRAS E ECONÔMICAS MENSAIS: UMA APLICAÇÃO DE MIDAS
}

\author{
Pedro Tonon Zuanazzi * \\ Flávio Augusto ZiegelmanN ${ }^{\dagger}$
}

\begin{abstract}
Resumo
A previsão do PIB é um dos principais balizadores para as decisões produtivas de agentes econômicos. Com o objetivo de realizar previsões para o crescimento do PIB trimestral brasileiro, são utilizadas 16 séries mensais financeiras e econômicas como potenciais preditores, abrangendo o período do segundo trimestre de 1996 ao quarto trimestre de 2012. Para isso, aplicaram-se as abordagens MIDAS (Mixed Data Sampling) e UMIDAS (Unrestricted Mixed Data Sampling), confrontando seus resultados de previsão fora da amostra com o benchmark ARMA. Foram encontrados erros de previsão menores nessas abordagens, principalmente quando utilizadas informações dentro do trimestre de previsão. Os resultados foram ainda melhores quando empregados múltiplos regressores.
\end{abstract}

Palavras-chave: Previsões; PIB trimestral; MIDAS; UMIDAS.

\begin{abstract}
The GDP forecast is an important indicator for production decisions taken by economic agents. In order to make forecasts for the Brazilian quarterly GDP growth, we used 16 monthly financial and economic series as potential predictors, covering the period from the second quarter of 1996 to the fourth quarter of 2012. For this purpose, we applied MIDAS (Mixed Data Sampling) and UMIDAS (Unrestricted Mixed Data Sampling) approaches and compared the out of sample forecasts with the benchmark ones provided by ARMA. MIDAS and UMIDAS showed smaller prediction errors, especially when information inside the quarter forecast is used. The results were even better when multiple regressors were employed.
\end{abstract}

Keywords: Forecast; Quarterly GDP; MIDAS; UMIDAS.

JEL classification: E00, G00.

DOI: http://dx.doi.org/10.1590/1413-8050/ea515

\footnotetext{
* (FEE e PPGA-UFRGS). E-mail: pedro@fee.tche.br

† Departamento de Estatística, (PPGA e PPGE — UFRGS). E-mail: flavioz@ufrgs.br
} 


\section{Introdução}

Previsões para o comportamento da economia são importantes desafios da moderna literatura de econometria de finanças, contribuindo para que agentes econômicos - públicos e privados - possam tomar decisões com menor incerteza. Do ponto de vista das empresas, prever melhor a renda futura dos agentes implica em decisões de investimento mais precisas, pois uma das variáveis que determina a função de demanda é a renda. Do ponto de vista dos governantes, a redução da incerteza da produção futura permite estimar melhor a arrecadação tributária.

Os preços dos ativos cotados na bolsa de valores são baseados nas expectativas de fluxos de caixas a serem gerados futuramente, descontados por uma taxa de retorno determinada pelos investidores (Williams 1938). Com base nisso, uma vez que as expectativas de lucros das empresas estão associadas às expectativas de variações macroeconômicas futuras, a perspectiva dos investidores sobre as alterações posteriores da economia impacta nos preços das ações no presente, implicando que os preços dos ativos podem ser utilizados para prever variações macroeconômicas futuras, como por exemplo do PIB (Stock \& Watson 2003). Mais precisamente, Grossman (1976) mostrou que, se não há custos de transação ou outras fricções financeiras no mercado, o preço atual de um ativo é a agregação perfeita de todas as previsões individuais do seu preço no dia seguinte. Assim, na medida em que os ativos financeiros são créditos sobre a produção futura, seus preços hoje fornecem informações sobre o futuro da economia.

A ideia de utilizar preços de ativos para prever cenários macroeconômicos surgiu com Mitchell \& Burns (1938), que incluíram o índice Dow Jones em sua lista de indicadores para prever expansões e contrações da economia norteAmericana. Mais recentemente, diversos estudos foram realizados a fim de confirmar essa hipótese, dentre eles destacam-se Stock \& Watson (2003), Tay (2007), Galvao (2007), Clements \& Galvao (2009), Andreou et al. (2013), Bessec (2011), Barsoum (2011), Guerin \& Marcellino (2013) e Ferrara \& Marsilli (2013), tendo sido encontradas evidências dessa relação.

Um dilema enfrentado por pesquisadores ao tentar identificar associações entre variáveis macroeconômicas e séries financeiras está no fato de elas serem divulgadas em frequências distintas. No Brasil, enquanto o PIB é divulgado trimestralmente, outras séries com potencial poder preditivo para o PIB são divulgadas em frequências mais altas, como por exemplo retornos de ativos que podem ser coletados inclusive intradiariamente.

Historicamente, a maneira mais usual para trabalhar com os dados era transformá-los para a mesma periodicidade, agregando as séries de maior frequência. Contudo, os métodos de agregação simples acabam por desperdiçar informações contidas nos dados. Algumas técnicas surgiram a fim de aperfeiçoar a solução para essa questão (ver Litterman 1984, Zadrozny 1988, Miller \& Chin 1996), sendo a abordagem MIDAS (Mixed Data Sampling), introduzida por Ghysels et al. (2007), ${ }^{1}$ uma alternativa muito utilizada na literatura recente.

MIDAS trata-se de uma proposta para relacionar dados em diferentes frequências sem a necessidade de desconsiderar parte da informação de uma ou mais séries (como nos casos de agregação simples). Os dados com frequências

\footnotetext{
${ }^{1}$ A primeira versão foi disponibilizada como working paper em 2002.
} 
mais altas são ponderados por uma função polinomial parcimoniosa capaz de adquirir diversos formatos com poucos parâmetros, não havendo um grande prejuízo no número de graus de liberdade. Desde sua introdução, a aplicação dessa técnica em trabalhos internacionais tem sido realizada em diferentes análises, dentre elas na relação entre variáveis macroeconômicas e séries financeiras. Tay (2007) aplicou MIDAS para prever o crescimento do PIB tendo como preditores somente retornos acionários diários, identificando uma melhor previsão na comparação com a alternativa de utilizar somente o retorno das ações no último dia de cada trimestre; Clements \& Galvao (2009) utilizaram MIDAS para prever a taxa de crescimento do PIB dos Estados Unidos, incorporando, entre outros indicadores, retornos acionários mensais, tendo encontrado melhores previsões, em alguns casos, do que os benchmarks AR e ADL; Andreou et al. (2013) utilizaram em torno de mil séries financeiras diárias (entre retornos de ativos, preços de commodities, taxas de câmbios e títulos do governo) para realizar previsões do PIB dos EUA, encontrando ganhos nas previsões; Barsoum (2011) realizou previsões para o crescimento do PIB norte-americano utilizando séries financeiras diárias, obtendo melhores previsões quando utilizadas informações dentro do trimestre de previsão; Ferrara \& Marsilli (2013) realizaram previsões para o PIB antes da grande recessão de 2008 para quatro países da zona do Euro (Alemanha, França, Itália e Espanha), tendo como preditores retornos diários de ativos, o preço do barril de petróleo e a diferença entre as taxas de juros de títulos de longo e curto prazos (termspread), encontrando significância estatística apenas para os retornos dos ativos. No Brasil, a abordagem MIDAS foi aplicada por Wink Junior \& Pereira (2011) e Santos \& Ziegelmann (2012) a fim de realizar previsões de volatilidade realizada, não tendo sido encontrada, em nossa revisão bibliográfica, o emprego dessa abordagem para previsões macroeconômicas.

Embora as funções polinomiais disponíveis para o emprego da abordagem MIDAS sejam bastante maleáveis, é possível que essa flexibilidade não seja sempre o suficiente para refletir o verdadeiro comportamento dos dados analisados. Uma alternativa simples é a utilização de um parâmetro para cada observação da variável de alta frequência. Essa abordagem, que já foi aplicada por Koenig et al. (2003) e Clements \& Galvao $(2008,2009)$, foi posteriormente denominada UMIDAS (Unrestricted Mixed Data Sampling) por Foroni et al. (2011). O maior prejuízo dessa abordagem está no fato de ela reduzir o número de graus de liberdade da estimação devido ao elevado número de parâmetros, principalmente quando a diferença de frequência entre a variável dependente e os regressores é grande, ou quando o número de defasagens utilizadas para os regressores é elevado.

O presente trabalho teve como objetivo realizar previsões para a variação do PIB trimestral brasileiro dessazonalizado em relação ao trimestre imediatamente anterior (com correção por sazonalidade), comparando as modelagens MIDAS, UMIDAS e o benchmark ARMA, tendo como regressores séries mensais (todas em variação): ativos cotados na Bovespa, índice Bovespa, índice Dow Jones, taxa de câmbio (preço do dólar PTAX) divulgada pelo BACEN, CDI (BACEN), produção industrial (geral, extrativa e de transformação) divulgada pelo IBGE, preço do barril do petróleo (EIA - U.S. Energy Information Administration) e valor total das exportações (MDIC). Para o ajuste do modelo foram utilizados dados da variação do PIB no período entre o $2^{\circ}$ trimestre de 1996 e o $4^{\circ}$ trimestre de 2012 . Foram realizadas previsões com as variáveis isoladas e com modelos múltiplos. Os resultados obtidos apresentaram 
ganhos de previsão fora da amostra um passo a frente na utilização de MIDAS sobre UMIDAS, que por sua vez apresentou melhores resultados do que a previsão pela técnica univariada ARMA. O emprego de MIDAS obteve resultados ainda melhores quando utilizados múltiplos regressores, e também, em alguns casos, quando utilizadas informações dos regressores mensais já ocorridas dentro do trimestre de previsão.

\section{Modelos para dados em diferentes frequências}

\subsection{Métodos de agregação simples}

Agregações temporais das variáveis de alta frequência para mais baixas frequências são muito usadas em trabalhos aplicados em diversas áreas. Os estudos de agregação temporal têm início com o artigo de Amemiya \& Wu (1972), que demonstraram que se uma variável original é gerada por um modelo AR de ordem $p$, então sua agregação por média segue um modelo AR de ordem $p$ com uma estrutura MA nos resíduos. Tiao (1972) e Amemiya \& Wu (1972) abordam a questão da perda de desempenho das previsões univariadas com $n$ pequeno devido à agregação. Da mesma forma, alternativas como interpolar a série de baixa frequência também são bastante utilizadas na prática. É possível verificar que os métodos de agregação simples são casos restritos das abordagens MIDAS e UMIDAS (como por exemplo ponderar igualitariamente todas as ocorrências do regressor em cada período $t$, o mesmo caso da média simples).

Dentre os métodos de agregação simples, uma alternativa bastante utilizada na prática é extrair a variação acumulada da variável de alta frequência durante cada ocorrência da variável de baixa frequência. Trata-se de um método propício no caso em que o regressor é uma variável de estoque. Seja $x_{t}^{(m)}$ a variável de alta frequência, $y_{t}$ a variável de baixa frequência e $m$ o número de vezes que $x$ se repete para cada ocorrência de $y_{t}$ em cada período $t$. Um método intuitivo e simples reside em utilizar a variação do nível de $x_{t}^{(m)}$ ao longo do período $t$. Por exemplo, na utilização do Ibovespa mensal para prever variações trimestrais do PIB, extrairíamos o valor do Ibovespa no final de cada trimestre $t$, pelo valor do Ibovespa no final de cada trimestre imediatamente anterior. Dessa forma teríamos:

$$
x_{t}^{*}=\frac{x_{t}^{(m)}}{x_{t-1}^{(m)}}-1,
$$

em que $x_{t}^{(m)}$ é a última observação de $x$ no período $t$. Por fim, a variação do PIB $\left(y_{t}\right)$ seria estimada através de um modelo de regressão simples tendo como regressor a variação defasada do Ibovespa:

$$
y_{t}=\beta_{0}+\beta_{1} x_{t-1}^{*}+\varepsilon_{t} .
$$

Outro método de agregação simples é extrair a média da variável de alta frequência, o que é adequado para os casos em que os regressores são variáveis de fluxo. Seu emprego reside em calcular a média das $m$ ocorrências de $x_{t}^{(m)}$ ao longo de $t$. Dessa maneira, temos para cada período $t$ : 


$$
\bar{X}_{t}=\frac{1}{m} \sum_{k=1}^{m} L^{\frac{(k-1)}{m}} x_{t}^{(m)},
$$

onde $L^{\frac{(k-1)}{m}} x_{t}^{(m)}=x_{t-\frac{(k-1)}{m}}^{(m)}$, sendo $m$ o número de vezes que a variável de alta frequência se repete em cada período $t$ e $x_{t-\frac{p}{m}}^{(m)}$ a $p$-ésima defasagem do regressor. Assim, a estimação de $y_{t}$ é dada por

$$
y_{t}=\beta_{0}+\beta_{1} \bar{X}_{t-1}+\varepsilon_{t}
$$

Assim como no caso da variação acumulada, no caso da média simples dados da variável de mais alta frequência (que podem conter informações importantes) são descartados, pois estamos utilizando a mesma ponderação para todas as ocorrências de $x_{t}^{(m)}$ no período $t$, não considerando que dados mais recentes possam ter pesos maiores (ou menores) na estimativa de $y_{t}$. De maneira geral, ao empregar um método de agregação simples obtemos perda informacional dos dados.

\subsection{As abordagens MIDAS e UMIDAS}

Embasados nos modelos ADLs (Adjusted Distributed Lag), Ghysels et al. (2007) (sendo seu primeiro working paper de 2002) e Ghysels et al. (2004) propuseram uma nova classe de modelos denominada MIDAS (Mixed Data Sampling), que permite que as variáveis dependentes e independentes estejam em frequências heterogêneas. Nesta abordagem, as variáveis explicativas (em mais alta frequência) são ponderadas por funções que com poucos parâmetros conseguem atingir boa flexibilidade. As mais comumente utilizadas são a função exponencial de Almon e a função polinomial Beta. Assim, os modelos MIDAS utilizam o potencial de toda a informação contida nos dados.

Como alternativa, a abordagem UMIDAS (Unrestricted Mixed Data Sampling) opta por estimar um parâmetro para cada defasagem da variável de alta frequência, ganhando ainda mais flexibilidade para os pesos das defasagens, mas com a desvantagem da proliferação do número de parâmetros. Tendo sido aplicada por Koenig et al. (2003) e Clements \& Galvao (2008, 2009), foi denominada UMIDAS posteriormente, por Foroni et al. (2011).

\section{MIDAS básico}

O modelo MIDAS possui uma estrutura lag polinomial envolvendo regressores com diferentes periodicidades. Em sua composição, a variável dependente $y_{t}$ apresenta uma ocorrência em cada unidade de tempo $t$, enquanto as variáveis independentes $x_{t}^{(m)}$, cujas frequências são iguais ou maiores que a da variável dependente, possuem $m$ observações para $t$. Por exemplo, se a frequência de $y_{t}$ for trimestral, $x_{t}^{(3)}$ seria a representação para dados mensais.

Conforme notação de Clements \& Galvao (2008), um modelo MIDAS com uma variável explicativa pode ser descrito por

$$
y_{t}=\beta_{0}+\beta_{1} B\left(L^{\frac{1}{m}} ; \theta\right) x_{t-1}^{(m)}+\varepsilon_{t},
$$


onde $B\left(L^{\frac{1}{m}} ; \theta\right)=\sum_{k=1}^{K} b(k ; \theta) L^{\frac{(k-1)}{m}}$ e $L^{\left(\frac{s}{m}\right)} x_{t-1}^{(m)}=x_{t-1-\frac{s}{m}}^{(m)}$. Nessa modelagem, $b(k$; $\theta$ ) representa uma função parcimoniosa de pesos, sendo $\theta$ o vetor de parâmetros dessa função e $\varepsilon_{t}$ um ruído branco. $B\left(L^{\frac{1}{m}} ; \theta\right)$ é um polinômio de grau $K$, sendo $K$ o número de defasagens de $x_{t}^{(m)}$ que impactam na previsão de $y_{t}$. Os parâmetros $\beta_{0}$, $\beta_{1}$ e $\theta$ são estimados por mínimos quadrados não lineares.

Como exemplo, suponhamos $m=3$ e $K=6$, onde $y_{t}$ é trimestral e $x_{t}^{(3)}$ representa o dado mensal. Nesse caso, a equação 5 pode ser escrita como

$$
y_{t}=\beta_{0}+\beta_{1} B\left(L^{\frac{1}{3}} ; \theta\right) x_{t-1}^{(3)}+\varepsilon_{t},
$$
onde $B\left(L^{1 / 3} ; \theta\right)=\sum_{k=1}^{6} b(k ; \theta) L^{\frac{(k-1)}{3}}$ e $L^{(s / 3)} x_{t-1}^{(3)}=x_{t-1-\frac{s}{3}}^{(3)}$. Como resultado, te-
mos:

$$
\begin{array}{r}
y_{t}=\beta_{0}+\beta_{1}\left[b(1 ; \theta) x_{t-1}^{(3)}+b(2 ; \theta) x_{t-1-\frac{1}{3}}^{(3)}+b(3 ; \theta) x_{t-1-\frac{2}{3}}^{(3)}+b(4 ; \theta) x_{t-2}^{(3)}+\right. \\
\left.b(5 ; \theta) x_{t-2-\frac{1}{3}}^{(3)}+b(6 ; \theta) x_{t-2-\frac{2}{3}}^{(3)}\right]+\varepsilon_{t} .
\end{array}
$$

Nesse caso, se estivermos prevendo o valor de $y_{t}$ para o primeiro trimestre de 2012, então $x_{t-1}^{(3)}$ seria o valor do regressor em dezembro de 2011, $x_{t-1-\frac{1}{3}}^{(3)}$ seria o valor de novembro de 2011, e assim por diante, até que $x_{t-2-\frac{2}{3}}^{(3)}$ seria o valor de julho de 2011 , o $6^{\circ}$ e último mês em que $x_{t}^{(m)}$ possui peso na previsão de $y_{t}$.

Enquanto a equação 5 é a fórmula para a previsão um passo a frente, de forma mais genérica, temos a previsão de $h$ passos a frente dada por

$$
y_{t}=\beta_{0}+\beta_{1} B\left(L^{\frac{1}{m}} ; \theta\right) x_{t-h}^{(m)}+\varepsilon_{t} .
$$

Em seu trabalho, Ghysels et al. (2007) discutem diversas alternativas para a especificação do polinômio $B\left(L^{\frac{1}{m}} ; \theta\right)$, sendo a função de defasagens exponencial de Almon (exponential Almon Lag), introduzida por Almon (1965), dada por

$$
b(k ; \theta)=\frac{e^{\theta_{1} k+\ldots+\theta_{Q} k^{Q}}}{\sum_{k=1}^{K} e^{\theta_{1} k+\ldots+\theta_{Q} k^{Q}}} .
$$

Dentre os benefícios dessa especificação, está o fato de que com apenas dois parâmetros $(Q=2)$ é possível obter diversos formatos para a função, além de que, dependendo dos valores dos parâmetros, é possível obter uma queda mais rápida ou mais lenta dos pesos conforme as defasagens aumentam. A Figura 1 faz simulações para diferentes valores dos parâmetros $\theta_{1}$ e $\theta_{2}$, considerando $K$ infinito (sendo $K$ o número de defasagens que impactam na previsão de $y_{t}$ ).

Com essa flexibilidade da função, supondo que o modelo esteja bem especificado, com apenas dois parâmetros além da constante $\beta_{0}$ e de $\beta_{1}$ é provável que as estimativas atinjam valores próximos aos verdadeiros pesos geradores dos dados aleatórios. ${ }^{2}$

\footnotetext{
${ }^{2}$ Outra função com propriedades satisfatórias é a função polinomial Beta, também comumente empregada na aplicação de MIDAS — ver Ghysels et al. (2007).
} 


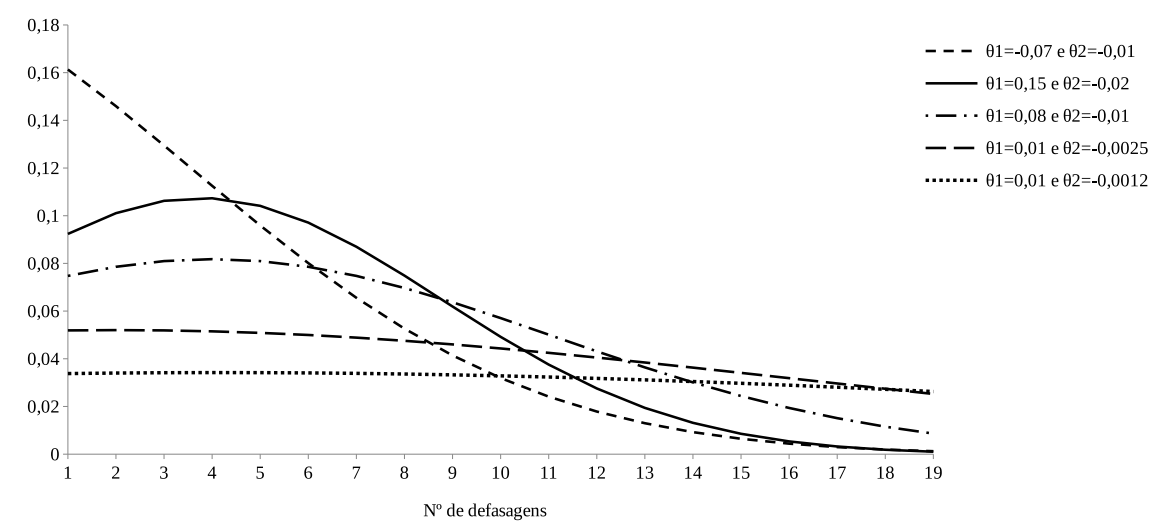

Fonte: Elaborado pelo autor.

Figura 1: Função de defasagens exponencial de Almon com 2 parâmetros e algumas de suas variações.

\section{Autorregressivo Midas (AR-MIDAS)}

A maneira mais intuitiva para incorporar defasagens de $y_{t}$, no modelo de previsão MIDAS com $h$ passos a frente, é dada por

$$
y_{t}=\beta_{0}+\sum_{i=1}^{P} \lambda_{i} y_{t-i}+\beta_{1} B\left(L^{\frac{1}{m}} ; \theta\right) x_{t-h}^{(m)}+\varepsilon_{t} .
$$

Contudo, Ghysels et al. (2007) expõem que essa especificação não é sempre apropriada, uma vez que ela força uma sazonalidade possivelmente fictícia em $x_{t}$. Dessa forma, essa especificação deveria ser utilizada apenas para dados com padrão sazonal definido. Clements \& Galvao (2008) propuseram uma solução para a questão, introduzindo um fator comum para eliminar o problema da sazonalidade:

$$
y_{t}=\beta_{0}+\lambda y_{t-h}+\beta_{1} B\left(L^{\frac{1}{m}} ; \theta\right)\left(1-\lambda L^{h}\right) x_{t-h}^{(m)}+\varepsilon_{t} .
$$

Em seu artigo, Clements \& Galvao (2008) explicam o algoritmo de estimação para a equação 11, utilizado no presente trabalho (Ver subsubseção 2.2). Por fim, podemos inserir múltiplos regressores para estimar $y_{t}$, obtendo, assim, a equação final do modelo M-AR-MIDAS com $n$ variáveis independentes:

$$
y_{t}=\beta_{0}+\lambda y_{t-h}+\sum_{i=1}^{n} \beta_{i} B\left(L^{\frac{1}{m}} ; \theta_{i}\right)\left(1-\lambda L^{h}\right) x_{i, t-h}^{(m)}+\varepsilon_{t} .
$$

\section{UMIDAS}

Uma alternativa à abordagem MIDAS é a utilização de um parâmetro para cada observação da variável de alta frequência, denominada UMIDAS. Assim como no caso MIDAS, o modelo UMIDAS leva em consideração toda a informação de $x_{t}^{(m)}$ para prever $y_{t}$, com a diferença de não restringir que os pesos para as defasagens do regressor estejam ligados por uma função polinomial que pode não ser flexível o suficiente para representar os verdadeiros pesos geradores dos dados aleatórios. Seu maior prejuízo está na proliferação do número de parâmetros, diminuindo consideravelmente o número de graus de 
liberdade da estimação, principalmente quando a diferença das frequências de $x_{t}^{(m)}$ e $y_{t}$ é elevada ( $m$ grande).

Ao contrário do método de agregação por média simples, que pressupõe peso igual para cada observação, a abordagem UMIDAS aloca parâmetros para cada observação de $x_{t}^{(m)}$ dentro de cada período $t$, de maneira que as observações de $x_{t}^{(m)}$ não tenham todas a mesma ponderação dependendo de sua defasagem. Assim, usando a mesma notação da subsubseção 2.2, o modelo UMIDAS com apenas um regressor é dado por

$$
y_{t}=\beta_{0}+\sum_{k=1}^{K} \beta_{k} L^{\frac{(k-1)}{m}} x_{t-1}^{(m)}+\varepsilon_{t} .
$$

Dessa forma, são estimados $K+1$ parâmetros para o modelo com $K$ defasagens. De maneira mais genérica, pode-se facilmente inserir mais de um regressor de alta frequência e também utilizar defasagens da variável dependente $y_{t}$. Assim temos o modelo M-AR-UMIDAS dado por

$$
y_{t}=\beta_{0}+\sum_{d=1}^{D} \lambda_{d} y_{t-d}+\sum_{v=1}^{V} \sum_{k=1}^{K} \beta_{v, k} L^{\frac{(k-1)}{m}} x_{v, t-1}^{(m)}+\varepsilon_{t},
$$

onde $V$ é o número de variáveis independentes no modelo e $\lambda_{d}$ é o parâmetro autorregressivo da $d$-ésima defasagem da variável dependente $y_{t}$.

Embora essa abordagem utilize toda a informação de $x_{t}^{(m)}$ para prever $y_{t}$ (diferentemente dos métodos de agregação simples), o elevado número de parâmetros a serem estimados impacta na diminuição do número de graus de liberdade na estimação, tornando sua aplicabilidade praticamente inviável para $m$ grande, principalmente em modelos multivariados com mais de uma variável independente.

\section{Estimação e seleção dos modelos MIDAS e UMIDAS}

Ghysels et al. (2004) mostraram que o modelo MIDAS pode ser estimado por Mínimos Quadrados Não Lineares (MQNL), por Máxima Verossimilhança (MV) e pelo Método dos Momentos Generalizados (MMG), sendo MQNL o mais comumente usado na literatura. No presente trabalho empregou-se o MQNL, sendo aplicados seis algoritmos de otimização numérica para as estimativas dos parâmetros: nlm, nlminb, BFGS, L-BFGS-B, Nelder-Mead e CG. ${ }^{3}$ Para cada estimação optou-se pelas estimativas cuja soma dos quadrados dos resíduos foram mínimas, independente do algoritmo que tenha atingido o menor valor. Foi empregada a função de defasagens exponencial de Almon (ver figura 1) para a aplicação de MIDAS.

Para a estimação do modelo AR-MIDAS (equação 11) aplicou-se o algoritmo proposto por Clements \& Galvao (2008): primeiramente são extraídos os resíduos $\left(\varepsilon_{t}\right)$ da estimação MIDAS básica (equação 5) e, posteriormente, é estimado um valor inicial para $\lambda\left(\hat{\lambda}_{0}\right)$ através da equação $\hat{\lambda}_{0}=\left(\sum \hat{\varepsilon}_{t-h}^{2}\right)^{-1} \sum \hat{\varepsilon}_{t} \hat{\varepsilon}_{t-h}$. Então são calculados $y_{t}^{*}=y_{t}-\hat{\lambda}_{0} y_{t-h}$ e $x_{t}^{*(m)}=x^{(m)}-\hat{\lambda}_{0} x_{t-h}^{(m)}$ para obter estimativas para os parâmetros $\beta$ e $\theta\left(\hat{\theta}_{1}\right)$ aplicando mínimos quadrados não lineares em $y_{t}^{*}=\beta_{0}+\beta_{1} B\left(L^{1 / 3}, \theta\right) x_{t}^{*(m)}+\varepsilon_{t}$. Extrai-se, então, uma nova estimativa para

\footnotetext{
${ }^{3}$ Para a realização das otimizações utilizou-se o pacote "optimx" do software estatístico R.
} 
$\lambda\left(\hat{\lambda}_{1}\right)$ através dos resíduos dessa regressão. Por fim, utilizando $\hat{\lambda}_{1}$ e $\hat{\theta}_{1}$ como valores iniciais para a otimização numérica, roda-se MQNL para obter $\hat{\lambda}$ e $\hat{\theta}$ que minimizem a soma de quadrados dos resíduos no modelo final (equação 11).

A estimativa do modelo UMIDAS, em contrapartida, pode ser realizada por Mínimos Quadrados Ordinários (MQO), o que torna o processo de estimação dos parâmetros instantâneo e simples, ao contrário do modelo MIDAS.

\section{Aplicação empírica para a previsão do PIB trimestral brasileiro}

Utilizando dados da variação do PIB brasileiro dessazonalizada em relação ao trimestre imediatamente anterior, no período entre o $2^{\circ}$ trimestre de 1996 e o $4^{\circ}$ trimestre de 2012, foram primeiramente testados 16 regressores ${ }^{4}$ mensais em separado, sendo eles: retornos das ações CMIG4, PETR4, BBDC4, BBAS3, AMBV4, USIM5 e VALE5 (como critério de liquidez foram utilizados todos os ativos que tiveram negociação na Bovespa em todos os dias úteis desde o início do plano real, em 30/06/1994), variação do índice Bovespa, variação do índice Dow Jones, variação da taxa de câmbio em reais perante o dólar (PTAX), variação do rendimento do CDI, variação dessazonalizada da produção industrial mensal ${ }^{5}$ (geral e separada em extrativa e de transformação), variação em dólar do preço do barril do petróleo (brent) e variação dessazonalizada em dólar do valor total das exportações. Posteriormente foram estimados modelos das classes MIDAS e UMIDAS com múltiplos regressores.

A Figura 2 apresenta a série histórica da variação do PIB trimestral brasileiro dessazonalizado. Pode-se perceber que a ocorrência de variações negativas foi algo comum no período, com a crise de 2008 facilmente identificada.

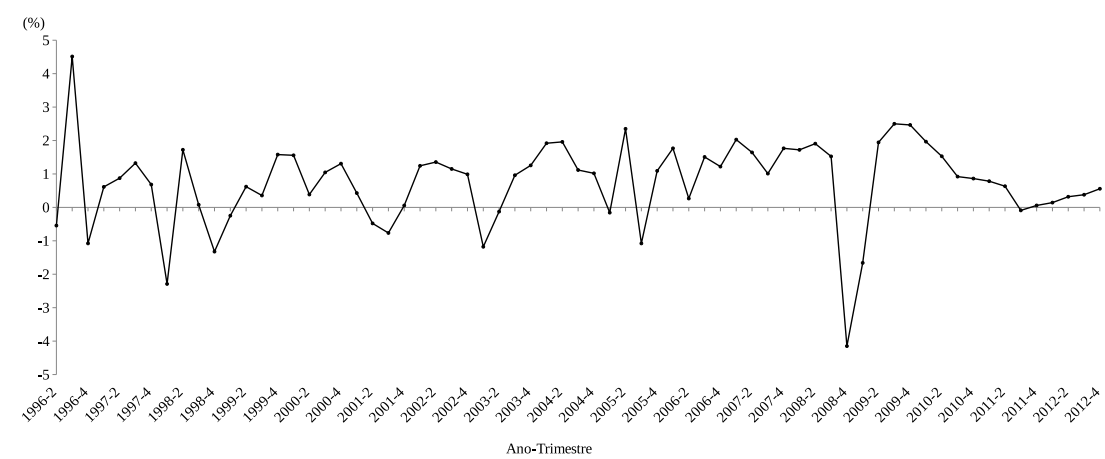

Fonte: IBGE.

Figura 2: Variação do PIB trimestral do Brasil sobre o trimestre imediatamente anterior, dessazonalizada, 1996-2 a 2012-4.

Tanto a série de variação do PIB quanto todos os regressores utilizados para a sua previsão tiveram a hipótese de raiz unitária rejeitada ao nível de

\footnotetext{
${ }^{4}$ Outros potenciais regressores mensais não foram empregados por não estarem disponíveis desde o começo do período utilizado, como as vendas do varejo (obtidas pela pesquisa mensal do comércio), o IBC-Br e o índice ABCR.

${ }^{5}$ A série é obtida pela pesquisa industrial mensal. Embora a pesquisa seja divulgada com mais de um mês de defasagem, ela é útil para a previsão do PIB, pois o mesmo é divulgado com aproximadamente dois meses de defasagem. Por exemplo, quando a PIM de fevereiro é divulgada em abril, o PIB do primeiro trimestre ainda não foi divulgado.
} 
5\% de significância, conforme os testes Dickey-Fuller aumentado (ver Said \& Dickey 1984) e Phillips-Perron (ver Phillips \& Perron 1988)). ${ }^{6}$ Dessa forma, nas estimativas realizadas no presente trabalho, as séries foram consideradas estacionárias.

Tanto para MIDAS quanto para UMIDAS foram realizadas, para cada trimestre de previsão, três estimativas em momentos diferentes de informação de $x_{t}^{(m)}$ : utilizando informações até o último mês do trimestre anterior ao de interesse (horizonte de previsão $h=1$ passo a frente), utilizando informações até o primeiro mês do trimestre de previsão ( $h=2 / 3$ passo a frente) e tendo informação até o segundo mês do trimestre de previsão ( $h=1 / 3$ passo a frente). A única diferença na estimação entre esses três momentos de previsão está no modo de como a matriz do(s) regressor(es) é organizada. Quando utilizado um mês dentro do trimestre de interesse $(h=2 / 3)$, o dado desse mês passa a ser a primeira defasagem de $x_{t}^{(m)}$ e o dado do último mês do trimestre anterior se torna a segunda defasagem. A Figura 3 apresenta exemplos de cada um dos três momentos, contendo números de defasagens arbitrários que poderiam ser utilizados para a previsão de um determinado trimestre (nesse exemplo o $3^{\circ}$ trimestre do ano II):

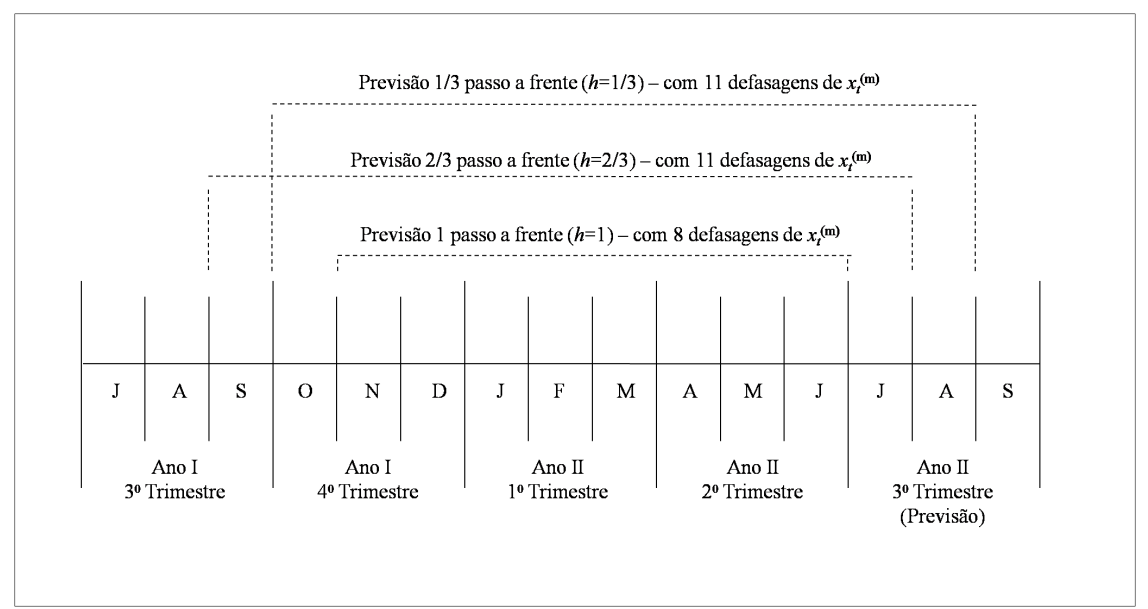

Fonte: Elaborado pelo autor.

Figura 3: Períodos de informação utilizados por um regressor mensal, 1, 2/3 e $1 / 3$ passo a frente, para a previsão do terceiro trimestre do ano II — datas e números de defasagens somente ilustrativos.

Para a definição do número de defasagens a ser utilizado em cada regressor, em cada horizonte $h$, optou-se, tanto para UMIDAS quanto para MIDAS, por escolher o de menor BIC. Nas estimativas por regressão múltipla, por simplicidade computacional, manteve-se o número de defasagens identificado para cada regressor na regressão simples.

Tanto nas estimativas por regressão múltipla quanto por regressão simples as previsões são realizadas para fora da amostra $-1,2 / 3$ e $1 / 3$ passo a frente - sendo que para cada nova informação de $y_{t}$ os parâmetros do modelo são novamente estimados, empregando, dessa maneira, previsões para 12 trimestres fora da amostra. Dessa forma, para os três horizontes de tempo

\footnotetext{
${ }^{6}$ Os valores-p de ambos os testes estão divulgados no Apêndice A.
} 
h, o primeiro e o último trimestres de previsão foram 2010:1 e 2012:4, tendo como amostras, para $h=1$, por exemplo, os períodos 1996:2 a 2009:4 e 1996:2 a 2012:3, respectivamente. Para $h=2 / 3$ utilizou-se, também, 1 mês dentro do próprio trimestre de previsão, e para $h=1 / 3$, utilizaram-se 2 meses dentro do trimestre de previsão. Posteriormente, foram comparadas as abordagens MIDAS, UMIDAS e ARMA pela raiz quadrada do erro quadrático médio da previsão (RMSFE - Root Mean Square Forecast Error).

\subsection{Previsões com regressores individuais}

Primeiramente, cada regressor foi utilizado isoladamente para a previsão da variação dessazonalizada do PIB trimestral do Brasil. A Tabela 1 apresenta os RMSFE dos 16 regressores, a razão entre o RMSFE encontrado por cada abordagem e o RMSFE obtido pelo ARMA de melhor ajuste (menor BIC) e o valor-p do teste de previsão de Diebold-Mariano $(\mathrm{DM})^{7}$ — ver Diebold \& Mariano (2002) — entre a previsão do modelo em questão e o modelo ARMA. Estão em asteriscos as razões que entre o RMSFE da estimativa em análise e o RMSFE do ARMA apresentaram valor abaixo de 0,7 , assim como os testes de Diebold-Mariano significantes ao nível de 10\%.

Os resultados, apresentados na Tabela 1, identificam boas previsões para diversos regressores na comparação com o ARMA, destacando-se: a variação do Ibovespa obteve RMSFE bem abaixo do ARMA tanto para UMIDAS quanto para MIDAS, com e sem a utilização de informação dentro do trimestre de previsão, tendo diferença significativa no teste DM ao nível de $10 \%$ para quase todos os modelos e horizontes de previsão testados; as produções da indústria (geral e somente de transformação) obtiveram os menores RMSFE nas previsões $2 / 3$ e $1 / 3$ passo a frente; a variação do valor total exportado apresentou boas previsões tanto para MIDAS quanto para UMIDAS, com os melhores resultados no horizonte de previsão $h=1$.

Para os regressores mencionados acima, com maiores destaques nas previsões fora da amostra (variações do Ibovespa, indústria geral, indústria de transformação e exportações), são apresentados abaixo os gráficos com seus pesos finais na abordagem MIDAS para cada defasagem. ${ }^{8}$

Quando analisados os pesos finais tendo como regressor a variação do Ibovespa (Figura 4), verifica-se sinal positivo para todas as defasagens, indicando que um crescimento do Ibovespa está associado a um crescimento posterior do PIB. Concomitantemente, percebe-se que o maior peso não é dado para a defasagem mais recente, principalmente na previsão $1 / 3$ passo a frente, indicando que as variações do Ibovespa de hoje já estão precificando a variação do PIB no futuro, e não no instante imediato.

Indo ao encontro dos resultados da Tabela 1 - de que as variações das produções das indústrias geral e de transformação obtiveram bons resultados quando utilizadas informações dentro do trimestre de previsão - verifica-se nas Figuras 5 e 6 que as curvas de pesos para $h=1 / 3$ possuem valores maiores nas duas primeiras defasagens (que fazem parte do trimestre de previsão), assim como as curvas de pesos para $h=2 / 3$, que possuem valores maiores para a

\footnotetext{
${ }^{7}$ A hipótese nula do teste bicaudal de Diebold-Mariano é que as duas estimativas possuem a mesma capacidade de previsão. Sua aplicação foi realizada com o pacote Forecast do software estatístico R.

${ }^{8}$ Os pesos finais são obtidos pela multiplicação do $\beta$ pelos valores extraídos com a curva exponencial de Almon de cada estimativa, conforme a equação 6 .
} 
Tabela 1: Comparação das abordagens MIDAS e UMIDAS com a ARMA para previsões da variação do PIB trimestral brasileiro, pelo RMSFE e pelo teste de Diebold-Mariano

\begin{tabular}{|c|c|c|c|c|c|c|c|c|c|c|c|c|c|}
\hline \multirow[t]{2}{*}{ Modelo } & \multirow[t]{2}{*}{ Horizonte (h) } & \multicolumn{3}{|c|}{ Índice BOVESPA } & \multicolumn{3}{|c|}{ CMIG4 } & \multicolumn{3}{|c|}{ PETR4 } & \multicolumn{3}{|c|}{ BBDC4 } \\
\hline & & $\begin{array}{l}\text { RMSFE } \\
(\%)\end{array}$ & $\begin{array}{l}\text { Razão ao } \\
\text { ARMA }\end{array}$ & $\begin{array}{l}\text { Teste DM } \\
\text { (valor-p) }\end{array}$ & $\begin{array}{l}\text { RMSFE } \\
(\%)\end{array}$ & $\begin{array}{l}\text { Razão ao } \\
\text { ARMA }\end{array}$ & $\begin{array}{l}\text { Teste DM } \\
\text { (valor-p) }\end{array}$ & $\begin{array}{l}\text { RMSFE } \\
(\%)\end{array}$ & $\begin{array}{l}\text { Razão ao } \\
\text { ARMA }\end{array}$ & $\begin{array}{l}\text { Teste DM } \\
\text { (valor-p) }\end{array}$ & $\begin{array}{l}\text { RMSFE } \\
(\%)\end{array}$ & $\begin{array}{l}\text { Razão ao } \\
\text { ARMA }\end{array}$ & $\begin{array}{l}\text { Teste DM } \\
\text { (valor-p) }\end{array}$ \\
\hline \multirow[t]{3}{*}{ UMIDAS } & 1 & 0,480 & $0,615^{*}$ & $0,098^{*}$ & 0,606 & 0,775 & 0,272 & 0,531 & $0,680^{*}$ & $0,096^{*}$ & 0,541 & $0,692^{*}$ & 0,100 \\
\hline & $2 / 3$ & 0,498 & $0,637^{*}$ & $0,078^{*}$ & 0,597 & 0,764 & 0,223 & 0,564 & 0,721 & 0,114 & 0,586 & 0,750 & 0,117 \\
\hline & $1 / 3$ & 0,499 & $0,638^{*}$ & $0,080^{*}$ & 0,592 & 0,757 & 0,220 & 0,602 & 0,770 & 0,193 & 0,690 & 0,882 & 0,480 \\
\hline \multirow[t]{3}{*}{ AR-UMIDAS } & 1 & 0,528 & $0,676^{*}$ & 0,194 & 0,632 & 0,809 & 0,404 & 0,587 & 0,751 & 0,256 & 0,577 & 0,738 & 0,202 \\
\hline & $2 / 3$ & 0,545 & $0,697^{*}$ & 0,179 & 0,606 & 0,775 & 0,326 & 0,682 & 0,872 & 0,526 & 0,620 & 0,793 & 0,285 \\
\hline & $1 / 3$ & 0,544 & $0,696^{*}$ & 0,190 & 0,594 & 0,760 & 0,335 & 0,647 & 0,828 & 0,396 & 0,699 & 0,895 & 0,632 \\
\hline \multirow[t]{3}{*}{ MIDAS } & 1 & 0,482 & $0,617^{*}$ & 0,102 & 0,606 & 0,775 & 0,271 & 0,720 & 0,922 & 0,521 & 0,553 & 0,708 & $0,080^{*}$ \\
\hline & $2 / 3$ & 0,432 & $0,553^{*}$ & $0,049^{*}$ & 0,670 & 0,858 & 0,452 & 0,759 & 0,971 & 0,783 & 0,565 & 0,723 & $0,022^{*}$ \\
\hline & $1 / 3$ & 0,495 & $0,633^{*}$ & $0,073^{*}$ & 0,668 & 0,855 & 0,382 & 0,779 & 0,997 & 0,975 & 0,541 & $0,692^{*}$ & $0,028^{*}$ \\
\hline \multirow[t]{3}{*}{ AR-MIDAS } & 1 & 0,575 & 0,736 & $0,095^{*}$ & 0,729 & 0,933 & 0,660 & 0,782 & 1,001 & 0,990 & 0,710 & 0,909 & 0,268 \\
\hline & $2 / 3$ & 0,485 & $0,620^{*}$ & $0,031^{*}$ & 0,809 & 1,035 & 0,823 & 0,887 & 1,136 & 0,235 & 0,927 & 1,186 & 0,337 \\
\hline & $1 / 3$ & 0,560 & 0,717 & $0,044^{*}$ & 0,771 & 0,986 & 0,924 & 0,746 & 0,954 & 0,763 & 0,684 & 0,875 & $0,086^{*}$ \\
\hline \multirow[t]{2}{*}{ Modelo } & Horizonte (h) & \multicolumn{3}{|c|}{ BBAS3 } & \multicolumn{3}{|c|}{ AMBV4 } & \multicolumn{3}{|c|}{ USIM5 } & \multicolumn{3}{|c|}{ VALE5 } \\
\hline & & $\begin{array}{l}\text { RMSFE } \\
(\%)\end{array}$ & $\begin{array}{l}\text { Razão ao } \\
\text { ARMA }\end{array}$ & $\begin{array}{l}\text { Teste DM } \\
\text { (valor-p) }\end{array}$ & $\begin{array}{l}\text { RMSFE } \\
(\%)\end{array}$ & $\begin{array}{l}\text { Razão ao } \\
\text { ARMA }\end{array}$ & $\begin{array}{l}\text { Teste DM } \\
\text { (valor-p) }\end{array}$ & $\begin{array}{l}\text { RMSFE } \\
(\%)\end{array}$ & $\begin{array}{l}\text { Razão ao } \\
\text { ARMA }\end{array}$ & $\begin{array}{l}\text { Teste DM } \\
\text { (valor-p) }\end{array}$ & $\begin{array}{l}\text { RMSFE } \\
(\%)\end{array}$ & $\begin{array}{l}\text { Razão ao } \\
\text { ARMA }\end{array}$ & $\begin{array}{l}\text { Teste DM } \\
\text { (valor-p) }\end{array}$ \\
\hline \multirow[t]{3}{*}{ UMIDAS } & 1 & 0,541 & $0,692^{*}$ & $0,097^{*}$ & 0,688 & 0,880 & 0,641 & 0,540 & $0,691^{*}$ & 0,222 & 0,555 & 0,711 & 0,289 \\
\hline & $2 / 3$ & 0,585 & 0,749 & 0,109 & 0,691 & 0,885 & 0,656 & 0,538 & $0,688^{*}$ & 0,191 & 0,557 & 0,713 & 0,290 \\
\hline & $1 / 3$ & 0,689 & 0,882 & 0,475 & 0,552 & 0,706 & 0,125 & 0,541 & $0,692^{*}$ & 0,206 & 0,561 & 0,717 & 0,300 \\
\hline \multirow[t]{3}{*}{ AR-UMIDAS } & 1 & 0,580 & 0,742 & 0,207 & 0,656 & 0,840 & 0,510 & 0,594 & 0,759 & 0,340 & 0,565 & 0,722 & 0,330 \\
\hline & $2 / 3$ & 0,619 & 0,792 & 0,279 & 0,658 & 0,842 & 0,517 & 0,605 & 0,774 & 0,337 & 0,566 & 0,724 & 0,329 \\
\hline & $1 / 3$ & 0,700 & 0,896 & 0,630 & 0,560 & 0,716 & 0,210 & 0,602 & 0,771 & 0,341 & 0,573 & 0,733 & 0,353 \\
\hline \multirow[t]{3}{*}{ MIDAS } & 1 & 0,553 & 0,707 & $0,077^{*}$ & 0,613 & 0,784 & 0,390 & 0,565 & 0,723 & 0,178 & 0,555 & 0,710 & 0,290 \\
\hline & $2 / 3$ & 0,573 & 0,734 & $0,052^{*}$ & 0,612 & 0,783 & 0,388 & 0,571 & 0,731 & 0,107 & 0,556 & 0,711 & 0,290 \\
\hline & $1 / 3$ & 0,543 & $0,695^{*}$ & $0,026^{*}$ & 0,611 & 0,782 & 0,386 & 0,596 & 0,763 & 0,119 & 0,555 & 0,711 & 0,290 \\
\hline \multirow[t]{3}{*}{ AR-MIDAS } & 1 & 0,712 & 0,911 & 0,266 & 0,794 & 1,016 & 0,953 & 0,672 & 0,859 & 0,396 & 0,474 & $0,606^{*}$ & $0,066^{*}$ \\
\hline & $2 / 3$ & 0,922 & 1,180 & 0,348 & 0,807 & 1,032 & 0,904 & 0,700 & 0,895 & 0,367 & 0,509 & $0,651^{*}$ & $0,052^{*}$ \\
\hline & $1 / 3$ & 0,706 & 0,903 & 0,198 & 0,834 & 1,067 & 0,800 & 0,714 & 0,914 & 0,395 & 0,515 & $0,659^{*}$ & $0,056^{*}$ \\
\hline
\end{tabular}


Tabela 1: Comparação das abordagens MIDAS e UMIDAS com a ARMA para previsões da variação do PIB trimestral brasileiro, pelo RMSFE e pelo teste de Diebold-Mariano (Continuação)

\begin{tabular}{|c|c|c|c|c|c|c|c|c|c|c|c|c|c|}
\hline \multirow[t]{2}{*}{ Modelo } & \multirow[t]{2}{*}{ Horizonte (h) } & \multicolumn{3}{|c|}{ Índice Dow Jones } & \multicolumn{3}{|c|}{ Barril do Petróleo } & \multicolumn{3}{|c|}{ CDI } & \multicolumn{3}{|c|}{ Dólar (PTAX) } \\
\hline & & $\begin{array}{l}\text { RMSFE } \\
(\%)\end{array}$ & $\begin{array}{l}\text { Razão ao } \\
\text { ARMA }\end{array}$ & $\begin{array}{l}\text { Teste DM } \\
\text { (valor-p) }\end{array}$ & $\begin{array}{l}\text { RMSFE } \\
(\%)\end{array}$ & $\begin{array}{l}\text { Razão ao } \\
\text { ARMA }\end{array}$ & $\begin{array}{l}\text { Teste DM } \\
\text { (valor-p) }\end{array}$ & $\begin{array}{l}\text { RMSFE } \\
(\%)\end{array}$ & $\begin{array}{l}\text { Razão ao } \\
\text { ARMA }\end{array}$ & $\begin{array}{l}\text { Teste DM } \\
\text { (valor-p) }\end{array}$ & $\begin{array}{l}\text { RMSFE } \\
(\%)\end{array}$ & $\begin{array}{l}\text { Razão ao } \\
\text { ARMA }\end{array}$ & $\begin{array}{l}\text { Teste DM } \\
\text { (valor-p) }\end{array}$ \\
\hline \multirow[t]{3}{*}{ UMIDAS } & 1 & 0,530 & $0,678^{*}$ & 0,177 & 0,571 & 0,730 & 0,169 & 0,824 & 1,055 & 0,783 & 0,506 & $0,647^{*}$ & 0,114 \\
\hline & $2 / 3$ & 0,653 & 0,836 & 0,373 & 0,661 & 0,845 & 0,370 & 0,832 & 1,065 & 0,759 & 0,572 & 0,732 & 0,159 \\
\hline & $1 / 3$ & 0,679 & 0,869 & 0,462 & 0,673 & 0,861 & 0,353 & 0,832 & 1,064 & 0,756 & 0,616 & 0,789 & 0,319 \\
\hline \multirow[t]{3}{*}{ AR-UMIDAS } & 1 & 0,552 & 0,707 & 0,288 & 0,647 & 0,828 & 0,509 & 0,908 & 1,162 & 0,509 & 0,501 & $0,641^{*}$ & 0,173 \\
\hline & $2 / 3$ & 0,624 & 0,799 & 0,372 & 0,697 & 0,892 & 0,650 & 0,919 & 1,176 & 0,502 & 0,576 & 0,737 & 0,253 \\
\hline & $1 / 3$ & 0,685 & 0,877 & 0,585 & 0,686 & 0,878 & 0,574 & 0,915 & 1,171 & 0,508 & 0,662 & 0,848 & 0,540 \\
\hline \multirow[t]{3}{*}{ MIDAS } & 1 & 0,658 & 0,842 & 0,510 & 0,603 & 0,772 & 0,305 & 0,791 & 1,012 & 0,954 & 0,503 & $0,643^{*}$ & 0,187 \\
\hline & $2 / 3$ & 0,686 & 0,878 & 0,548 & 0,617 & 0,790 & 0,294 & 0,783 & 1,002 & 0,994 & 0,590 & 0,755 & 0,323 \\
\hline & $1 / 3$ & 0,514 & 0,658 & 0,182 & 0,659 & 0,844 & 0,368 & 0,779 & 0,997 & 0,990 & 0,530 & $0,678^{*}$ & 0,173 \\
\hline \multirow{3}{*}{ AR-MIDAS } & 1 & 0,647 & 0,828 & 0,539 & 0,763 & 0,976 & 0,874 & 0,821 & 1,050 & 0,797 & 0,567 & 0,726 & 0,213 \\
\hline & $2 / 3$ & 0,749 & 0,959 & 0,833 & 0,712 & 0,912 & 0,585 & 0,831 & 1,063 & 0,777 & 0,778 & 0,996 & 0,983 \\
\hline & $1 / 3$ & 0,479 & $0,613^{*}$ & 0,202 & 0,677 & 0,867 & 0,398 & 0,828 & 1,059 & 0,784 & 0,669 & 0,856 & 0,351 \\
\hline \multirow[t]{2}{*}{ Modelo } & Horizonte $(\mathrm{h})$ & \multicolumn{3}{|c|}{ Indústria Geral } & \multicolumn{3}{|c|}{ Indústria Extrativa } & \multicolumn{3}{|c|}{ Indústria de Transformação } & \multicolumn{3}{|c|}{ Exportações } \\
\hline & & $\begin{array}{l}\text { RMSFE } \\
(\%)\end{array}$ & $\begin{array}{l}\text { Razão ao } \\
\text { ARMA }\end{array}$ & $\begin{array}{l}\text { Teste DM } \\
\text { (valor-p) }\end{array}$ & $\begin{array}{l}\text { RMSFE } \\
(\%)\end{array}$ & $\begin{array}{l}\text { Razão ao } \\
\text { ARMA }\end{array}$ & $\begin{array}{l}\text { Teste DM } \\
\text { (valor-p) }\end{array}$ & $\begin{array}{l}\text { RMSFE } \\
(\%)\end{array}$ & $\begin{array}{l}\text { Razão ao } \\
\text { ARMA }\end{array}$ & $\begin{array}{l}\text { Teste DM } \\
\text { (valor-p) }\end{array}$ & $\begin{array}{l}\text { RMSFE } \\
(\%)\end{array}$ & $\begin{array}{l}\text { Razão ao } \\
\text { ARMA }\end{array}$ & $\begin{array}{l}\text { Teste DM } \\
\text { (valor-p) }\end{array}$ \\
\hline \multirow[t]{3}{*}{ UMIDAS } & 1 & 0,686 & 0,877 & 0,559 & 0,645 & 0,826 & 0,385 & 0,601 & 0,770 & 0,394 & 0,498 & $0,637^{*}$ & 0,177 \\
\hline & $2 / 3$ & 0,529 & $0,677^{*}$ & 0,137 & 0,519 & $0,664^{*}$ & 0,128 & 0,488 & $0,624^{*}$ & 0,188 & 0,581 & 0,743 & 0,206 \\
\hline & $1 / 3$ & 0,491 & $0,628^{*}$ & 0,120 & 0,638 & 0,816 & 0,376 & 0,473 & $0,605^{*}$ & 0,169 & 0,619 & 0,791 & 0,238 \\
\hline \multirow{3}{*}{ AR-UMIDAS } & 1 & 0,631 & 0,807 & 0,378 & 0,620 & 0,793 & 0,341 & 0,570 & 0,729 & 0,331 & 0,450 & $0,575^{*}$ & 0,158 \\
\hline & $2 / 3$ & 0,507 & $0,649^{*}$ & 0,119 & 0,521 & $0,667^{*}$ & 0,179 & 0,461 & $0,590^{*}$ & 0,157 & 0,584 & 0,747 & 0,277 \\
\hline & $1 / 3$ & 0,495 & $0,634^{*}$ & 0,176 & 0,688 & 0,881 & 0,614 & 0,470 & $0,601^{*}$ & 0,176 & 0,581 & 0,744 & 0,270 \\
\hline \multirow[t]{3}{*}{ MIDAS } & 1 & 0,601 & 0,769 & 0,308 & 0,655 & 0,838 & 0,296 & 0,553 & 0,708 & 0,282 & 0,460 & $0,588^{*}$ & 0,141 \\
\hline & $2 / 3$ & 0,421 & $0,538^{*}$ & $0,093^{*}$ & 0,637 & 0,815 & 0,231 & 0,488 & $0,624^{*}$ & 0,188 & 0,460 & $0,588^{*}$ & 0,141 \\
\hline & $1 / 3$ & 0,407 & $0,521^{*}$ & 0,103 & 0,550 & 0,704 & 0,149 & 0,411 & $0,526^{*}$ & 0,119 & 0,525 & $0,672^{*}$ & 0,247 \\
\hline \multirow[t]{3}{*}{ AR-MIDAS } & 1 & 0,638 & 0,816 & 0,304 & 0,506 & $0,648^{*}$ & 0,207 & 0,593 & 0,759 & 0,286 & 0,504 & $0,645^{*}$ & 0,187 \\
\hline & $2 / 3$ & 0,390 & $0,499^{*}$ & 0,120 & 0,618 & 0,791 & 0,180 & 0,455 & $0,582^{*}$ & 0,180 & 0,504 & $0,645^{*}$ & 0,187 \\
\hline & $1 / 3$ & 0,403 & $0,516^{*}$ & 0,104 & 0,595 & 0,761 & 0,184 & 0,404 & $0,517^{*}$ & 0,118 & 0,570 & 0,729 & 0,359 \\
\hline
\end{tabular}

Os RMSFE foram calculados através dos erros de previsão 1, 2/3 e 1/3 passo a frente, estimando novamente os parâmetros para cada nova observação do PIB trimestral. Todos os regressores estão em variação e foram utilizados isoladamente. Estão destacados em asteriscos $\left(^{*}\right)$ as razões dos RMSFE em relação ao ARMA menores que 0,7 , assim como os testes de Dibold-Mariano significantes ao nível de $10 \%$.

Fonte: Elaborado pelo autor. 


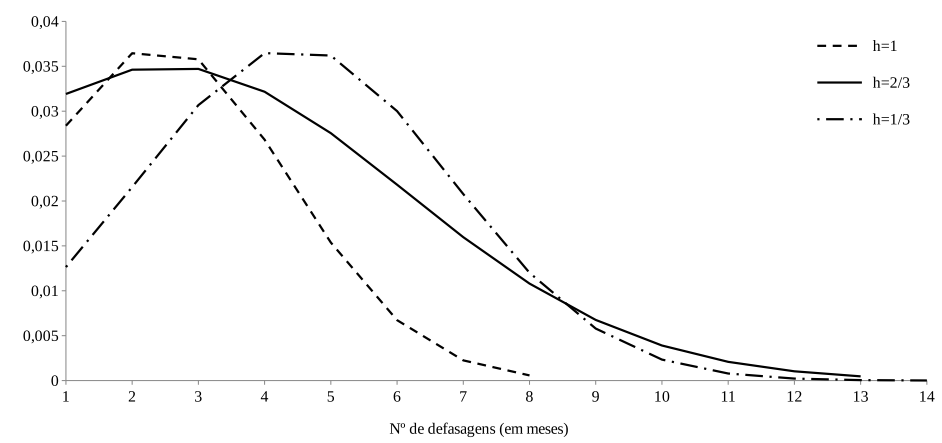

Fonte: Elaborado pelo autor.

* Os pesos representam o quanto a variação de uma unidade do regressor, em cada defasagem, impacta na variação do PIB.

Figura 4: Distribuição dos pesos obtidos pela estimativa MIDAS nas previsões $1,2 / 3$ e 1/3 passo a frente com somente um regressor: variação do Ibovespa

primeira defasagem (único mês dentro do trimestre de previsão nesses casos). Esses resultados são coerentes, uma vez que a indústria compõe o PIB.

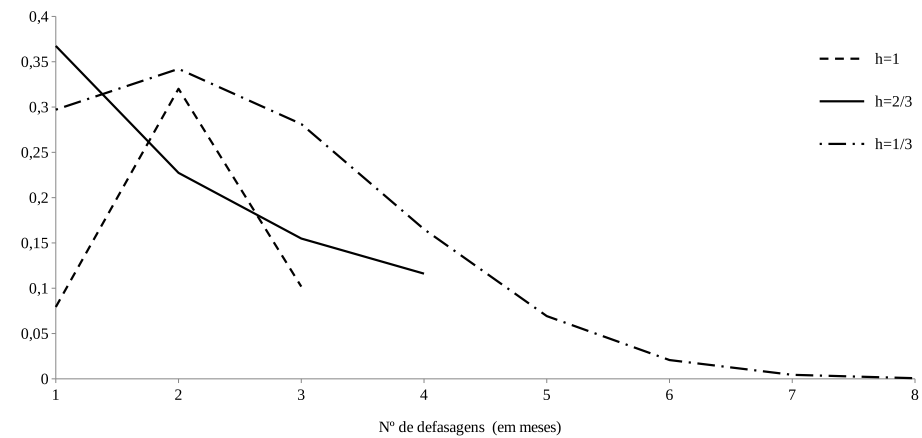

Fonte: Elaborado pelo autor.

* Os pesos representam o quanto a variação de uma unidade do regressor, em cada defasagem, impacta na variação do PIB.

Figura 5: Distribuição dos pesos* obtidos pela estimativa MIDAS nas previsões $1,2 / 3$ e $1 / 3$ passo a frente com somente um regressor: variação da produção da indústria geral.

A Figura 7 apresenta os pesos obtidos tendo como regressor a variação das exportações (dessazonalizada e em dólar). Pode ser identificada uma relação negativa, com pesos maiores no penúltimo e no antepenúltimo mês do trimestre anterior ao da previsão.

Os resultados dessa subseção - que identificam, em geral, uma superioridade do modelo MIDAS em relação ao benchmark ARMA, principalmente com a utilização do Ibovespa e da produção da indústria - convergem com os obtidos por Guerin \& Marcellino (2013), ${ }^{9}$ que também encontraram menores RMSFE, relativamente ao benchmark $\operatorname{AR}(1)$, ao utilizar séries mensais

\footnotetext{
${ }^{9}$ Os autores, além de compararem o modelo MIDAS com o benchmark AR(1), introduzem a abordagem Markov switching MIDAS.
} 


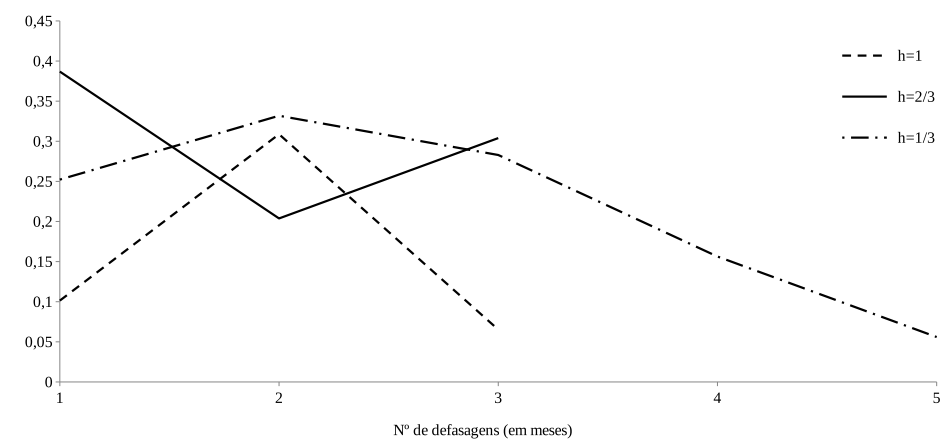

Fonte: Elaborado pelo autor.

* Os pesos representam o quanto a variação de uma unidade do regressor, em cada defasagem, impacta na variação do PIB.

Figura 6: Distribuição dos pesos* obtidos pela estimativa MIDAS nas previsões $1,2 / 3$ e $1 / 3$ passo a frente com somente um regressor: variação da produção da indústria de transformação

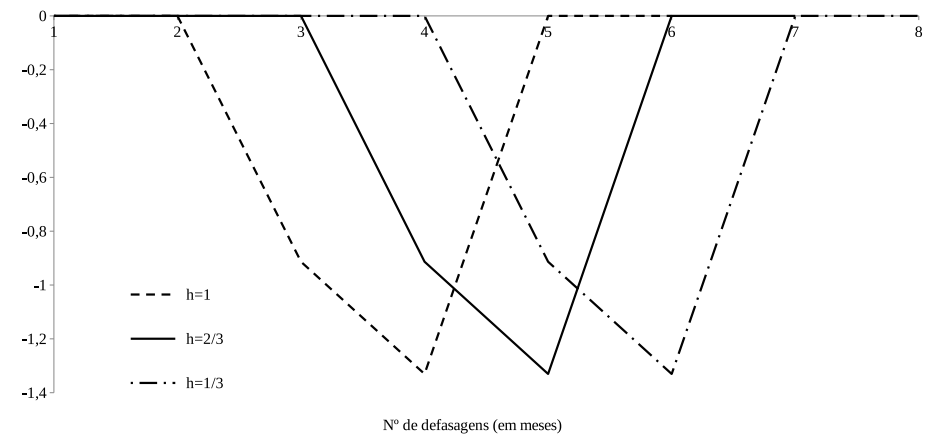

Fonte: Elaborado pelo autor.

* Os pesos representam o quanto a variação de uma unidade do regressor, em cada defasagem, impacta na variação do PIB.

Figura 7: Distribuição dos pesos* obtidos pela estimativa MIDAS nas previsões $1,2 / 3$ e $1 / 3$ passo a frente com somente um regressor: variação do valor das exportações 
do S\&P500 e da produção da indústria para as previsões do PIB dos Estados Unidos, sobretudo com o uso de informações dentro do trimestre de previsão. São similares, também, os resultados obtidos por Clements \& Galvao (2008), com baixos RMSFE em relação ao modelo AR ao utilizar a produção industrial mensal como preditor para o PIB norte-americano.

\subsection{Previsões com múltiplos regressores}

Testando diferentes combinações dos regressores, com o uso dos erros de previsão individuais, foram geradas previsões para o crescimento do PIB trimestral através dos modelos M-MIDAS, M-AR-MIDAS (equação 12), M-UMIDAS e M-AR-UMIDAS (equação 14). Foi escolhido um modelo final ${ }^{10}$ para a classe MIDAS e um modelo final para a classe UMIDAS para cada horizonte de previsão (1, 2/3 e 1/3 passo a frente), conforme retrata a Tabela 2. Na comparação entre os RMSFE das duas abordagens, tem-se que a classe MIDAS teve pior desempenho quando $h=1$, e melhor desempenho quando $h=2 / 3$ e $1 / 3$.

O menor RMSFE obtido, dentre todos os modelos e horizontes, foi pela estimação M-MIDAS com $h=1 / 3$ : um valor de 0,312 - o que representa uma razão de 0,399 sobre o RMSFE do ARMA. Em todos os horizontes, tanto para a classe MIDAS quanto para a UMIDAS, o Ibovespa fez parte do melhor modelo de previsão, enquanto que a produção da indústria geral ou a produção da indústria de transformação estiveram presentes quando utilizadas informações dentro do trimestre de previsão. ${ }^{11}$

De maneira geral, os resultados podem ser considerados satisfatórios, uma vez que os RMSFE encontrados estiveram muito abaixo do ARMA, sobretudo para os horizontes $h=1 / 3$ e 2/3, situações em que obteve-se significância estatística no teste DM ao nível de $10 \%$ através da classe MIDAS. Além disso, os melhores modelos múltiplos em cada classe, em qualquer horizonte de previsão, atingiram RMSFE menores do que todas as variáveis isoladas.

A Figura 8 contém os pesos obtidos pelo modelo M-MIDAS com as duas variáveis que formaram o modelo de menor RMSFE um passo a frente: variação do Ibovespa e variação do dólar. Ao passo que a relação entre o Ibovespa e o PIB se mostrou positiva (da mesma forma de quando esse regressor foi utilizado individualmente - Figura 4), a relação se mostrou negativa entre o dólar e o PIB: um aumento do preço do dólar está associado a uma posterior diminuição do PIB. Uma hipótese para essa relação é que quando a expectativa futura é de crescimento da economia há um fluxo de capitais internacionais que valorizam o real frente ao dólar no presente e, em contrapartida, quando a expectativa futura é de recessão, há uma fuga de investimentos internacionais, desvalorizando o real. Outro ponto importante está no fato de que o maior impacto que a variação do câmbio gera na previsão do crescimento do PIB ocorre sete meses antes do trimestre de previsão.

Quando analisamos os regressores finais estimados no modelo M-AR-MIDAS $2 / 3$ passo a frente temos que variações da produção da indústria geral estão associadas aos maiores impactos na variação do PIB, principalmente no mês mais recente (que pertence ao trimestre de previsão), o que era esperado pois

\footnotetext{
${ }^{10}$ empregou-se, no modelo final, o número de regressores que minimizasse o RMSFE para cada horizonte de previsão - realizando diferentes combinações das variáveis - e utilizou-se os números ótimos de defasagens obtidos individualmente para cada regressor via BIC.

${ }^{11}$ As produções da indústria geral e da indústria de transformação possuem autocorrelação de 0,941 , como pode ser verificado no apêndice $A$.
} 
Tabela 2: Comparação das abordagens MIDAS e UMIDAS com a ARMA, por regressão múltipla, para a previsão do crescimento do PIB trimestral brasileiro, pelo RMSFE e pelo teste de Diebold-Mariano

\begin{tabular}{|c|c|c|c|c|c|c|c|c|}
\hline \multirow[t]{2}{*}{ Horizonte (h) } & \multicolumn{4}{|c|}{ Classe MIDAS } & \multicolumn{3}{|c|}{ Classe UMIDAS } & \multirow[b]{2}{*}{ (valor-p) } \\
\hline & Melhor previsão & RMSFE (\%) & Razão ao ARMA & Melhor previsão & RMSFE (\%) & Razão ao ARMA & Teste DM & \\
\hline $1^{*}$ & M-MIDAS & 0,436 & $0,558^{* * * *}$ & 0,111 & M-AR-UMIDAS & 0,383 & $0,490^{* * * *}$ & 0,113 \\
\hline $2 / 3^{* *}$ & M-AR-MIDAS & 0,346 & $0,443^{* * * *}$ & $0,089^{* * * *}$ & M-AR-UMIDAS & 0,443 & $0,567^{* * * *}$ & 0,142 \\
\hline $1 / 3^{* * *}$ & M-MIDAS & 0,312 & $0,399^{* * * *}$ & $0,066^{* * * * *}$ & M-UMIDAS & 0,433 & $0,553^{* * * *}$ & 0,106 \\
\hline
\end{tabular}

${ }^{*}$ M-MIDAS: variações Ibovespa e dólar; M-AR-UMIDAS: variações Ibovespa, dólar e exportações.

** M-AR-MIDAS: variações Ibovespa, barril do petróleo e produção da indústria geral; M-AR-UMIDAS: variações Ibovespa e produção da indústria de transformação.

*** M-MIDAS: variações Ibovespa e produção da indústria geral; M-UMIDAS: variações Ibovespa e produção da indústria geral.

Os RMSFE foram calculados através dos erros de previsão $1,2 / 3$ e 1/3 passo a frente, estimando novamente os parâmetros para cada nova observação do PIB trimestral. Todos os regressores estão em variação e foram utilizados isoladamente. Estão destacados em asteriscos $\left.{ }^{(* * * *}\right)$ as razões dos RMSFE em relação ao ARMA menores que 0,7 , assim como os testes de Dibold-Mariano significantes ao nível de $10 \%$. Fonte: Elaborado pelo autor. 


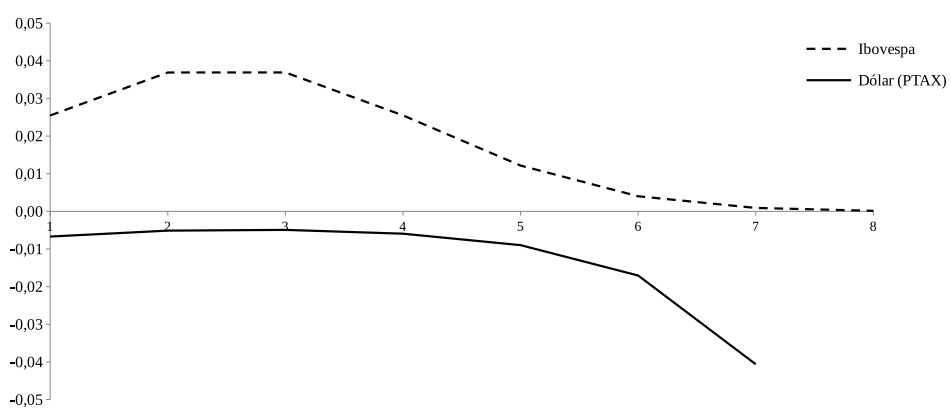

№ de defasagens (em meses)

Fonte: Elaborado pelo autor.

* Os pesos representam o quanto a variação de uma unidade do regressor, em cada defasagem, impacta na variação do PIB.

Figura 8: Distribuição dos pesos* obtidos pela estimativa MIDAS com melhor RMSFE no horizonte $h=1$

a indústria é um dos setores que compõe o PIB. Também fizeram parte do modelo final os regressores variação do Ibovespa e variação do barril do petróleo, porém com pesos pequenos.

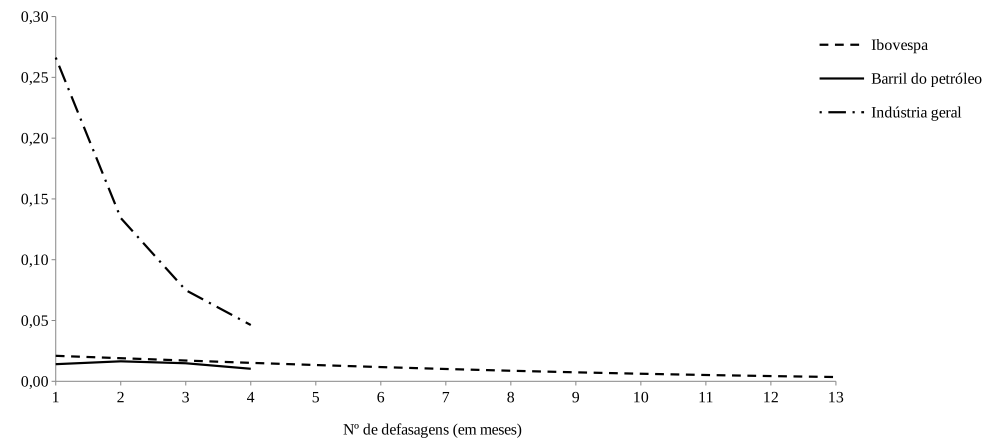

Fonte: Elaborado pelo autor.

* Os pesos representam o quanto a variação de uma unidade do regressor, em cada defasagem, impacta na variação do PIB.

Figura 9: Distribuição dos pesos* obtidos pela estimativa MIDAS com melhor RMSFE no horizonte $h=2 / 3$

Por fim, no modelo M-MIDAS 1/3 passo a frente, a produção da indústria geral também apresentou os maiores pesos para a estimativa do PIB trimestral, com valores maiores nos dois meses que fazem parte do trimestre de previsão e também no último mês do trimestre anterior (Figura 10). Também entraram na regressão múltipla, com pesos pequenos, o índice Bovespa e o índice Dow Jones.

\section{Conclusões}

Previsões para o comportamento da economia auxiliam na tomada de decisões de agentes econômicos em ambientes de incerteza. Com base nisso, o 


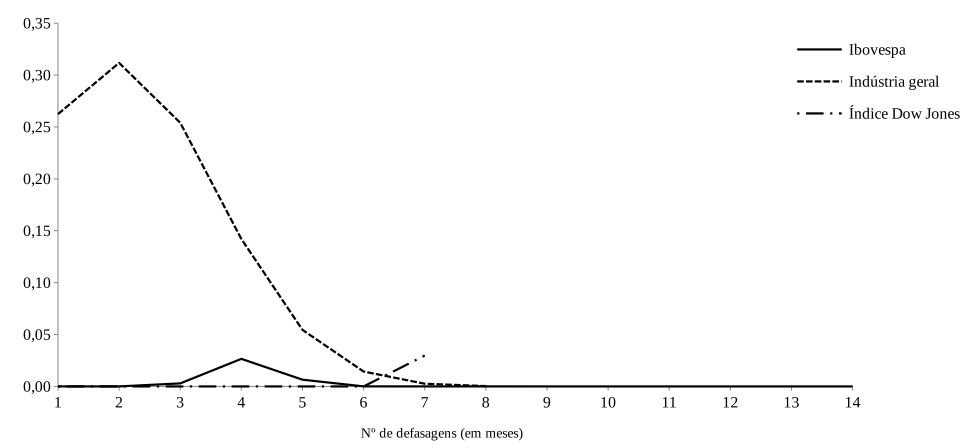

Fonte: Elaborado pelo autor.

* Os pesos representam o quanto a variação de uma unidade do regressor, em cada defasagem, impacta na variação do PIB.

Figura 10: Distribuição dos pesos ${ }^{*}$ obtidos pela estimativa MIDAS com melhor RMSFE no horizonte $h=1 / 3$

objetivo desse trabalho foi realizar previsões para o crescimento do PIB trimestral brasileiro (dessazonalizado). Para isso foram utilizadas, como preditores, séries financeiras e econômicas mensais. Para trabalhar com os dados nas diferentes frequências (trimestral e mensal), aplicaram-se os métodos MIDAS, AR-MIDAS, UMIDAS e AR-UMIDAS, tanto com cada regressor isoladamente quanto com modelos múltiplos, sendo os resultados de previsão comparados (por RMSFE) com o benchmark ARMA. Foram testados os horizontes de previsão 1, 2/3 e 1/3 passo a frente (nos dois últimos casos são utilizadas informações dentro do trimestre de previsão).

Os resultados apresentaram, para diversos regressores individuais, bons desempenhos nas previsões fora da amostra. Destacaram-se as variações do Ibovespa, das produções das indústrias geral e de transformação (principalmente quando utilizadas informações dentro do trimestre de previsão) e do valor das exportações. Esses resultados convergem com os obtidos por Clements \& Galvao (2008) e por Guerin \& Marcellino (2013) para a previsão do PIB dos Estados Unidos. Quando utilizados múltiplos regressores as previsões foram ainda melhores do que quando utilizadas variáveis isoladas.

Esse trabalho, de acordo com nossa revisão bibliográfica, possui o mérito de ser o primeiro a utilizar a abordagem MIDAS para previsões macroeconômicas no Brasil. Outros passos, no entanto, podem ser dados na tentativa de aperfeiçoar as previsões, como utilizar variáveis em nível ao invés de em variação (empregando cointegração - ver (Miller 2012)), aplicar a técnica de Markov Switching nos parâmetros MIDAS (ver Guerin \& Marcellino 2013), utilizar conjuntos maiores de dados realizando as previsões com a redução em fatores (ver Frale \& Monteforte 2011), entre outros.

\section{Agradecimentos}

Os autores agradecem a Jéfferson Augusto Colombo, João Frois Caldeira, Liane Werner, Marcelo Savino Portugal, Marcos Vinicio Wink Junior e Mariana Bartels pelas contribuições e sugestões. O autor Flavio Augusto Ziegelmann é Pesquisador CNPq (processo 305290/2012-6). Financiado parcialmente pelo 
CNPq (processo 485561/2013-1) e FAPERGS (processo 1994/12-6).

\section{Referências Bibliográficas}

Almon, S. (1965), 'The distributed lag between capital appropriations and expenditures', Econometrica: Journal of the Econometric Society 33, 178-196.

Amemiya, T. \& Wu, R. Y. (1972), 'The effect of aggregation on prediction in the autoregressive model', Journal of the American Statistical Association 67, 628-632.

Andreou, E., Ghysels, E. \& Kourtellos, A. (2013), 'Should macroeconomic forecasters use daily financial data and how?', Journal of Business E Economic Statistics 31(2), 240-251.

Barsoum, F. (2011), How useful is financial market data in forecasting uk real gdp? Working Paper.

Bessec, M. (2011), Forecasting gdp growth with a markov-switching factor midas model. Working Paper.

Clements, M. P. \& Galvao, A. B. (2008), 'Macroeconomic forecasting with mixed-frequency data: Forecasting output growth in the united states', Journal of Business E Economic Statistics 26(4), 546-554.

Clements, M. P. \& Galvao, A. B. (2009), 'Forecasting us output growth using leading indicators: An appraisal using midas models', Journal of Applied Econometrics 24(7), 1187-1206.

Diebold, F. X. \& Mariano, R. S. (2002), 'Comparing predictive accuracy', Journal of Business E economic statistics 20(1), 134-144.

Ferrara, L. \& Marsilli, C. (2013), 'Financial variables as leading indicators of gdp growth: Evidence from a midas approach during the great recession', Applied Economics Letters 20(3), 233-237.

Foroni, C., Marcellino, M. \& Schumacher, C. (2011), U-midas: Midas regressions with unrestricted lag polynomials. Working Paper, Deutsche Bundesbank, Research Centre.

Frale, C. \& Monteforte, L. (2011), Famidas: a mixed frequency factor model with midas structure. working paper, Bank of Italy Temi di Discussione.

Galvao, A. B. (2007), Changes in predictive ability with mixed frequency data. Working Paper, Department of Economics, Queen Mary, University of London.

Ghysels, E., Santa-Clara, P. \& Valkanov, R. (2004), The midas touch: Mixed data sampling regression models. Working Paper.

Ghysels, E., Sinko, A. \& Valkanov, R. (2007), 'Midas regressions: Further results and new directions', Econometric Reviews 26(1), 53-90.

Grossman, S. (1976), 'On the efficiency of competitive stock markets where trades have diverse information', The Journal of Finance 31(2), 573-585. 
Guerin, P. \& Marcellino, M. (2013), 'Markov-switching midas models', Journal of Business E Economic Statistics 31(1), 45-56.

Koenig, E. F., Dolmas, S. \& Piger, J. (2003), 'The use and abuse of real-time data in economic forecasting', Review of Economics and Statistics 85(3), 618628.

Litterman, R. B. (1984), 'Above-average national growth in 1985 and 1986', Federal Reserve Bank of Minneapolis Quarterly Review 8(4), 3-7.

Miller, J. I. (2012), Cointegrating midas regressions and a midas test, in 'Symposium on Empirical Finance and Financial Econometrics'.

Miller, P. \& Chin, D. (1996), 'Using monthly data to improve quarterly model forecasts', Federal Reserve Bank of Minneapolis Quarterly Review 20(2), 16-33.

Mitchell, W. C. \& Burns, A. F. (1938), Statistical indicators of cyclical revivals, National Bureau of Economic Research, Bulletin 69.

Phillips, P. C. B. \& Perron, P. (1988), 'Testing for a unit root in time series regression', Biometrika 75(2), 335-346.

Said, E. \& Dickey, D. A. (1984), 'Testing for unit roots in autoregressivemoving average models of unknown order', Biometrika 71(3), 599-607.

Santos, D. G. \& Ziegelmann, F. A. (2012), Multi-period volatility predictions: A comparative study using midas regressions. thirty-fourth Meeting of the Brazilian Econometric Society.

Stock, J. H. \& Watson, M. W. (2003), 'Forecasting output and inflation: The role of asset prices', Journal of Economic Literature 41(3), 788-829.

Tay, A. (2007), Financial variables as predictors of real output growth. Working Paper, SMU Economics \& Statistics.

Tiao, G. C. (1972), 'Asymptotic behaviour of temporal aggregates of time series', Biometrika 59(3), 525-531.

Williams, J. B. (1938), The theory of investment value, Harvard University Press.

Wink Junior, M. V. \& Pereira, P. L. V. (2011), 'Modeling and forecasting of realized volatility: Evidence from brazil', Brazilian Review of Econometrics 31(2), 315-337.

Zadrozny, P. (1988), 'Gaussian likelihood of continuous-time armax models when data are stocks and flows at different frequencies', Econometric Theory $4(1), 108-124$.

\section{Apêndice A}


Tabela A.1: Matriz de correlação dos regressores* utilizados para a previsão do PIB trimestral brasileiro

\begin{tabular}{|c|c|c|c|c|c|c|c|c|}
\hline & Índice BOVESPA & CMIG4 & PETR4 & BBDC4 & BBAS3 & AMBV4 & USIM5 & VALE5 \\
\hline Indice BOVESPA & 1,0000 & 0,6869 & 0,8011 & 0,7120 & 0,7152 & 0,5785 & 0,6297 & 0,5474 \\
\hline CMIG4 & 0,6869 & 1,0000 & 0,5635 & 0,5294 & 0,5141 & 0,4490 & 0,3961 & 0,3018 \\
\hline PETR4 & 0,8011 & 0,5635 & 1,0000 & 0,5816 & 0,5888 & 0,4143 & 0,4866 & 0,3667 \\
\hline BBDC4 & 0,7120 & 0,5294 & 0,5816 & 1,0000 & 0,9909 & 0,4779 & 0,4239 & 0,3198 \\
\hline BBAS3 & 0,7152 & 0,5141 & 0,5888 & 0,9909 & 1,0000 & 0,4812 & 0,4201 & 0,3235 \\
\hline AMBV4 & 0,5785 & 0,4490 & 0,4143 & 0,4779 & 0,4812 & 1,0000 & 0,3162 & 0,3864 \\
\hline USIM5 & 0,6297 & 0,3961 & 0,4866 & 0,4239 & 0,4201 & 0,3162 & 1,0000 & 0,3582 \\
\hline VALE5 & 0,5474 & 0,3018 & 0,3667 & 0,3198 & 0,3235 & 0,3864 & 0,3582 & 1,0000 \\
\hline Índice Dow Jones & 0,6238 & 0,4016 & 0,4909 & 0,4299 & 0,4325 & 0,4006 & 0,4487 & 0,3550 \\
\hline Barril do petróleo & 0,1538 & 0,1411 & 0,3012 & 0,0107 & 0,0112 & $-0,0907$ & 0,2021 & 0,1884 \\
\hline CDI & $-0,0027$ & 0,0860 & 0,0832 & 0,0066 & 0,0053 & $-0,0730$ & $-0,1034$ & $-0,0677$ \\
\hline Dólar (PTAX) & $-0,2562$ & $-0,2178$ & $-0,2929$ & $-0,2869$ & $-0,2934$ & 0,0907 & $-0,3327$ & 0,3183 \\
\hline Indústria geral & 0,0751 & 0,0483 & $-0,0153$ & 0,0410 & 0,0255 & 0,0371 & 0,0784 & 0,1144 \\
\hline Indústria extrativa & 0,0252 & $-0,0306$ & $-0,0151$ & 0,0658 & 0,0564 & 0,0009 & 0,0714 & 0,0348 \\
\hline Indústria de transformação & 0,0690 & 0,0563 & $-0,0191$ & $-0,0010$ & $-0,0131$ & 0,0428 & 0,0662 & 0,1306 \\
\hline Exportações & $-0,0421$ & 0,0540 & $-0,0681$ & 0,0272 & 0,0243 & $-0,0500$ & $-0,0130$ & $-0,1224$ \\
\hline
\end{tabular}

${ }^{*}$ Todos os regressores estão em variação.

Fonte: Elaborado pelo autor. 
Tabela A.1: Matriz de correlação dos regressores* utilizados para a previsão do PIB trimestral brasileiro (continuação)

\begin{tabular}{|c|c|c|c|c|c|c|c|c|}
\hline & $\begin{array}{l}\text { Índice } \\
\text { Dow Jones }\end{array}$ & $\begin{array}{l}\text { Barril do } \\
\text { petróleo }\end{array}$ & CDI & $\begin{array}{l}\text { Dólar } \\
\text { (PTAX) }\end{array}$ & $\begin{array}{l}\text { Indústria } \\
\text { geral }\end{array}$ & $\begin{array}{l}\text { Indústria } \\
\text { extrativa }\end{array}$ & $\begin{array}{l}\text { Indústria de } \\
\text { transformação }\end{array}$ & Exportações \\
\hline Índice BOVESPA & 0,6238 & 0,1538 & $-0,0027$ & $-0,2562$ & 0,0751 & 0,0252 & 0,0690 & $-0,0421$ \\
\hline CMIG4 & 0,3018 & 0,4016 & 0,1411 & 0,0860 & $-0,2178$ & 0,0483 & $-0,0306$ & 0,0540 \\
\hline BBDC4 & 0,4299 & 0,0107 & 0,0066 & $-0,2869$ & 0,0410 & 0,0658 & $-0,0010$ & 0,0272 \\
\hline BBAS3 & 0,4325 & 0,0112 & 0,0053 & $-0,2934$ & 0,0255 & 0,0564 & $-0,0131$ & 0,0243 \\
\hline AMBV 4 & 0,4006 & $-0,0907$ & $-0,0730$ & 0,0907 & 0,0371 & 0,0009 & 0,0428 & $-0,0500$ \\
\hline Índice Dow Jones & 1,0000 & 0,0913 & 0,0385 & $-0,3101$ & 0,0455 & 0,0273 & 0,0316 & $-0,0611$ \\
\hline Barril do petróleo & 0,0913 & 1,0000 & 0,0828 & $-0,1333$ & 0,1405 & 0,0006 & 0,1649 & $-0,0610$ \\
\hline CDI & 0,0385 & 0,0828 & 1,0000 & $-0,0175$ & $-0,0506$ & $-0,0650$ & $-0,1041$ & 0,1504 \\
\hline Dólar (PTAX) & $-0,3101$ & $-0,1333$ & $-0,0175$ & 1,0000 & 0,0210 & 0,0034 & 0,0632 & $-0,0812$ \\
\hline Indústria geral & 0,0455 & 0,1405 & $-0,0506$ & 0,0210 & 1,0000 & 0,4437 & 0,9410 & $-0,0202$ \\
\hline Indústria extrativa & 0,0273 & 0,0006 & $-0,0650$ & 0,0034 & 0,4437 & 1,0000 & 0,2343 & $-0,0076$ \\
\hline
\end{tabular}

\footnotetext{
* Todos os regressores estão em variação.
}

Fonte: Elaborado pelo autor. 
Tabela A.2: Valores-p dos testes de raiz unitária Dickey-Fuller aumentado e Phillips-Perron para o PIB trimestral e seus regressores

\begin{tabular}{lcc}
\hline & DF aumentado (valor-p) & PP $($ valor-p) \\
\hline PIB trimestral & $<0,01$ & $<0,01$ \\
Índice BOVESPA & $<0,01$ & $<0,01$ \\
CMIG4 & $<0,01$ & $<0,01$ \\
PETR4 & $<0,01$ & $<0,01$ \\
BBDC4 & $<0,01$ & $<0,01$ \\
BBAS3 & $<0,01$ & $<0,01$ \\
AMBV4 & $<0,01$ & $<0,01$ \\
USIM5 & $<0,01$ & $<0,01$ \\
VALE5 & $<0,01$ & $<0,01$ \\
Indice Dow Jones & $<0,01$ & $<0,01$ \\
Barril do petróleo & $<0,01$ & $<0,01$ \\
CDI & $<0,01$ & $<0,01$ \\
Dólar (PTAX) & $<0,01$ & $<0,01$ \\
Indústria geral & $<0,01$ & $<0,01$ \\
Indústria extrativa & $<0,01$ & $<0,01$ \\
Indústria de transformação & $<0,01$ & $<0,01$ \\
Exportações & 0,039 & $<0,01$ \\
\hline
\end{tabular}

Todas as variáveis estão em variação.

Fonte: Elaborado pelo autor. 